\title{
Versatility of Amantadine and Rimantadine for Detection of Cancer
}

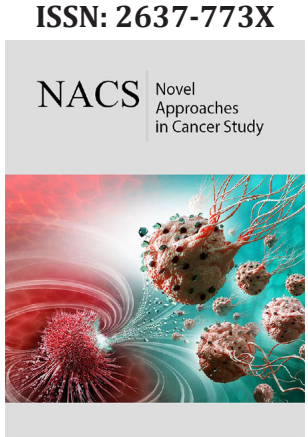

*Corresponding author: Paramjit S Tappia, Asper Clinical Research Institute \& Office of Clinical Research, Winnipeg, MB, R2H 2A6, Canada

Submission: 輩 April 16, 2020

Published: 此April 21, 2020

Volume 4 - Issue 4

How to cite this article: Bram Ramjiawan, Andrew W Maksymiuk, Rashid Ahmed Bux, Paramjit S Tappia, et al. Versatility of Amantadine and Rimantadine for Detection of Cancer. Nov Appro in Can Study. 4(4). NACS.000591. 2020. DOI: 10.31031/NACS.2020.04.000591

Copyright@ Paramjit S Tappia. This article is distributed under the terms of the Creative Commons Attribution 4.0 International License, which permits unrestricted use and redistribution provided that the original author and source are credited.
Bram Ramjiawan ${ }^{1,2}$, Andrew W Maksymiuk ${ }^{3,4}$, Paramjit S Tappia ${ }^{1 *}$, Rashid Ahmed Bux ${ }^{5}$, Parveen S Akhtar ${ }^{6}$, Donald Miller ${ }^{2}$ and Daniel S Sitar ${ }^{2,4}$

${ }^{1}$ Asper Clinical Research Institute \& Office of Clinical Research, Canada

${ }^{2}$ Department of Pharmacology \& Therapeutics, Rady Faculty of Health Sciences, Canada

${ }^{3}$ Cancer Care Manitoba, Canada

${ }^{4}$ Department of Internal Medicine, Canada

${ }^{5}$ BioMark Diagnostics Inc., Canada

${ }^{6}$ Department of Medical Oncology, Bangladesh

\section{Introduction}

Globally, an estimated 9.6 million people died from different types of cancer in 2017; in other words, every sixth death in the world was due to cancer, second only to cardiovascular diseases. The total number of cancer deaths continues to increase. In fact, by 2030 , the global burden of cancer is expected to be 21.7 million new cases and 13 million cancer deaths [1]. On Feb 4 2020, the World Health Organization (WHO) stated the need to step up cancer services in low and middle-income countries. WHO warned that, if current trends continue, the world will see a $60 \%$ increase in cancer cases over the next two decades. The greatest increase (an estimated $81 \%$ ) in new cases will occur in low- and middle-income countries, where survival rates are currently the lowest [2]. Early detection and diagnosis of cancer can lead to timely therapeutic/surgical interventions that can increase the chances of survival.

\section{The Problem}

Despite intense research and discovery over the years of many candidate biomarkers for cancer screening, only a few have transitioned to routine use in the clinic. Promising candidates include circulating tumor cells and cell-free tumor DNA, miRNAs, exosomes, secretome of the gut microbiota, exhaled volatile organic compounds, proteomics and metabolomics (liquid biopsy). These face many challenges before routine implementation in the clinical setting. The uptake and utilization of low-dose CT scans as a screening tool has been disappointing, partly because of the cost, perceived risk of exposure to ionizing radiation, and high proportion of false positives. Thus, the availability of an inexpensive, accurate, reproducible and simple test would therefore be of clinical and economic benefit.

\section{Our Approach}

Spermidine/spermine N1-acetyltransferase-1 (SSAT-1) is involved in the homeostasis of the polycationic aliphatic amines, spermine and spermidine. These polyamines are intimately involved in cell growth, proliferation and cell death [3]. The upregulation of SSAT-1 in different types of cancer cells is well documented. We have developed a simple test that evaluates SSAT1 activity by measuring acetylated products in the urine. On this basis, we believe that SSAT-1 activity can predict the presence of cancer and possibly disease progression. Our test is based on oral administration of a single dose of a US FDA-approved drug amantadine. Amantadine is an approved prophylactic drug used against influenza A infection and for treatment of Parkinson's disease. The standard dose is $100-200 \mathrm{mg}$ per day. Amantadine is excreted almost entirely unchanged by the kidney with a plasma half-life of 12-14 hours in persons with a creatinine clearance greater than $80 \mathrm{ml} / \mathrm{min}$. We have conducted several human excretion studies in subjects as old as 90 years of age without apparent adverse events.

Amantadine has been demonstrated to be a specific substrate of SSAT-1 [3], which is acetylated by the enzyme to produce acetylamantadine (AA), a terminal and stable end 
product excreted in urine [4,5]. We have developed a highly sensitive, accurate and reproducible method for measuring urinary levels of AA by liquid chromatography-tandem mass spectrometry (LC-MS/MS) technique. A US patent was granted in December of 2004, with the claim that excretion of AA in urine will serve as a diagnostic screening test for cancer in humans [6]. An ELISA point of care system using monoclonal antibodies is under development. Recently, we have reported the clinical utility of amantadine to detect elevated SSAT-1 activity by measuring increased concentration of AA in the urine of cancer patients. This increase was supported by the increase of the SSAT- 1 gene transcripts and protein contents in patient-derived tumor tissue [7]. While high levels of SSAT-1 gene and protein expression were measured in human primary breast, prostate, brain and lung tumor tissues, an increase in the urinary concentration of AA in cancer patients was observed [7]. We have further optimized urine collection time points for AA through pharmacokinetic/bioequivalence studies and additional validation of the use of amantadine as a mean to detect increased SSAT-1 activity [8]. The maximum concentration of the SSAT-1 metabolite AA in biological fluid samples (blood and urine) occurred 2-4 hours after an oral dose of $200 \mathrm{mg}$ of amantadine. These studies found no effect of ingestion of food on SSAT-1 activity.

In our earlier studies [8], although SSAT-1 was activated in patients with cancer, a number of ostensibly healthy adult volunteers had higher than expected urine AA concentration. We therefore, examined this outlier group by conducting a follow-up of urine analysis of AA as well as performing clinical assessments and additional blood analyses. In some of the outlier controls, higher than expected AA concentration was linked to increased serum carcinoembryonic antigen (a marker for cancer). We also observed that other clinical and radiographic assessments revealed underlying abnormalities in other cases that could represent premalignant conditions [9]. We believe that urine concentration of AA, after a one-time administration of $200 \mathrm{mg}$ amantadine, could be used as a simple and useful test for screening of cancer in high-risk populations.

For advanced-stage malignant disease, systemic chemotherapy is one of the main choices for therapy. The objectives are usually palliative in intent: i.e., to maintain or improve symptoms and quality of life, with the additional benefit of improving the survival duration - though falling short of cure in most cases. Evaluation of treatment response is often challenging as it may require several months to assess tumor regression using conventional techniques of physical examination, radiographic studies and/or conventional laboratory biochemistry studies. Serum tumor markers provide early indications, but are not available for many common types of cancer, e.g. lung cancer. Therefore, many patients may endure sideeffects of therapy for several months before clinicians are able to determine if therapy will be effective in achieving the intended therapeutic outcomes. With our amantadine test, we have also undertaken a pilot study designed to assess earlier and accurate response to systemic therapy. This response to treatment study examined the possibility that we can detect a therapeutic response by a change in the excretion of AA before it can be detected by conventional patient assessment methods. We are currently generating preliminary data that will hopefully support our contention that an early indication of a response to treatment may change the therapeutic approach and regimen. Three biological fluids will be analyzed, namely, urine, saliva and plasma.

We also wish to mention rimantadine (INN, sold under the trade name Flumadine) another orally administered antiviral drug used to treat, and in rare cases prevent, influenza virus A infection. Both rimantadine and amantadine are derivates of adamantane. Rimantadine was approved by the US Food and Drug Administration (FDA) in 1994. Rimantadine, like its antiviral cousin amantadine, also possesses some NMDA antagonistic properties and is used in the treatment of Parkinson's disease. Although, it should be noted that in general, neither rimantadine nor amantadine is a preferred agent for this therapy and would be reserved for cases of the disease that are less responsive to frontline treatments. In. comparison, amantadine and rimantadine as substrates, have very comparable SSAT- 1 affinity $(\mathrm{Km}$ in $\mu \mathrm{M}$ : 1659 for amantadine vs. 1835 for rimantadine), due to their similar chemical structures. Surprisingly, the maximum rate of reaction of SSAT-1 for rimantadine as substrate is almost 200fold higher than that of amantadine (Vmax expressed in pmol/ $\mathrm{min} /$ million cells, 0.364 rimantadine vs 0.00197 amantadine) and this makes rimantadine a better candidate than amantadine for the purpose of SSAT-1 diagnostics (all else assumed being equal clinical dose, ADME, toxicology, etc). In other words, if the clinical pharmacokinetics [10] of rimantadine is similar to amantadine, and that it can be acetylated in humans following a clinical dose, we will likely be able to detect much higher levels of rimantadine $\mathrm{N}$-acetylated metabolite in urine and plasma. In theory, with higher intracellular distribution of rimantadine and with increased SSAT-1 activity, a higher signal-to-noise ratio in detecting changes during SSAT- 1 enzyme regulation would be anticipated. These aspects are currently under investigation by our group. Lastly, since most brain tumors, especially glioblastoma, are not found until after symptoms appear, the ability of both amantadine and rimantadine to cross the blood-brain barrier provides a potential application of our test in the diagnosis and assessment of brain tumor malignancy. We are currently examining amantadine and rimantadine as drug biomarkers for brain tumor applications.

\section{Conclusion}

The determination of urinary (and plasma) AA concentration (and acetylated rimantadine) can be developed as a clinical test for:

a) detecting/diagnosing the presence of cancer;

b) determining the risk of cancer;

c) use as a treatment monitoring tool and

d) possible measurement of recurrence in cancer survivors (once baselines are determined post clinical clearance is provided). 


\section{References}

1. Global Cancer Facts \& Figures (2018) www.cancer.org/research/cancerfacts-statistics/global.html

2. World Health Organization (2020) https://www.who.int/news-room/ detail/04-02-2020-who-outlines-steps-to-save-7-million-lives-fromcancer.

3. Pegg AE (2016) Functions of polyamines in mammals. J Biol Chem 291(29): 1490-14912.

4. Bras AP, Hoff HR, Aoki FY, Sitar DS (1998) (Amantadine acetylation may be effected by acetyltransferases other than NAT1 or NAT2. Can J Physiol Pharmacol 76(7-8): 701-706.

5. Bras APM, Jänne J, Porter CW, Sitar DS (2001) Spermidine/spermine N1acetyltransferase catalyzes amantadine acetylation. Drug Metab Dispos 29(5): 676-680.
6. Sitar DS, Bras AP (2004) Method for assaying non-spermine/spermidine activity of spermidine/spermine $\mathrm{N}^{1}$-acetyl transferase (SSAT), USA.

7. Maksymiuk AW, Sitar DS, Ahmed R, Cheng B, Bach H, et al. (2018) Spermidine/spermine N1-acetyltransferase-1 (SSAT-1) as a diagnostic biomarker in human cancer. Future Sci OA 4(10): FSO345.

8. Maksymiuk AW, Tappia PS, Sitar DS, Akhtar PS, Khatun N, et al. (2018) Use of amantadine as substrate for SSAT-1 activity as a reliable clinical diagnostic assay for breast and lung cancer. Future Sci OA 5(2): FSO365.

9. Tappia PS, Maksymiuk AW, Sitar DS, Akhtar PS, Khatun N, et al. (2019) Predictive value and clinical significance of increased SSAT-1 activity in healthy adults. Future Sci OA 5(7): FSO400.

10. Hayden FG, Minocha A, Spyker DA, Hoffman HE (1985) (Comparative single-dose pharmacokinetics of amantadine hydrochloride and rimantadine hydrochloride in young and elderly adults. Antimicrob Agents Chemother 28(2): 216-221. 